\title{
Analysis Dataset Domain
}

National Cancer Institute

\section{Source}

National Cancer Institute. Analysis Dataset Domain. NCI Thesaurus. Code C49563.

A subject domain utilized for the submission of information encompassing and representing data, vocabulary or records related to the analysis of a dataset. 